\title{
O Impacto do protocolo de desmame de traqueostomia em pacientes vítimas de Traumatismo Cranioencefálico internados no Hospital Metropolitano de Urgência e Emergência no Pará
}

\author{
Fabiano José da Silva BOULHOSA ${ }^{1}$ \\ Leonardo Ramos Nicolau da COSTA ${ }^{2}$ \\ Gabriela Martins de LIMA ${ }^{3}$ \\ Kéven Lorena de Paula GONÇALVES ${ }^{4}$ \\ Juliana Anézia Rodrigues do AMARAL ${ }^{5}$ \\ Paola Katherine Esteves da SILVA ${ }^{6}$ \\ Patricia Gazel PICANÇO \\ Rafaela Cordeiro de MACÊDO ${ }^{8}$ \\ Renato da Costa TEIXEIRA ${ }^{9}$ \\ Sâmia da Cruz Albuquerque FEIO ${ }^{10}$
}

\begin{abstract}
${ }^{1}$ Especialista, preceptor do Programa de Residência Multiprofissional em Saúde em Urgência e Emergência no Trauma - Universidade do Estado do Pará; email: fabiano.boulhosa@gmail.com

${ }^{2}$ Mestre, coordenador do Programa de Residência Multiprofissional em Saúde em Urgência e Emergência no Trauma Universidade do Estado do Pará; email: leorcosta@gmail.com

${ }^{3}$ Especialista, coordenadora do setor de reabilitação do Hospital Metropolitano de Urgência e Emergência; email: gabimlima@gmail.com

${ }^{4}$ Especialista, fisioterapeuta do Hospital Metropolitano de Urgência e Emergência; email: keven.lorena@ hotmail.com

${ }^{5}$ Especialista; email:julianadoamaral@ymail.com

${ }^{6}$ Especialista, fisioterapeuta residente do Programa de Residência Multiprofissional em Saúde em Urgência e Emergência no Trauma- Universidade do Estado do Pará; email: paolaestevessilva@yahoo.com

${ }^{7}$ Fisioterapeuta residente do Programa de Residência Multiprofissional em Saúde em Urgência e Emergência no Trauma- Universidade do Estado do Pará; email: patrícia_gazel@ hotmail.com

${ }^{8}$ Especialista, preceptora do Programa de Residência Multiprofissional em Saúde em Urgência e Emergência no Trauma - Universidade do Estado do Pará; email: Rafaela_cmacedo@hotmail.com

${ }^{9}$ Doutor, professor Adjunto III da Universidade do Estado do Pará; email: teixeirarenato@globo.com

${ }^{10}$ Especialista; email: samiafeio@hotmail.com
\end{abstract}

Recebido em: 17/04/2015 - Aprovado em: 25/08/2015 - Disponibilizado em: 30/10/2015

\begin{abstract}
RESUMO: Esta pesquisa surgiu da vivência em um Hospital de referência em Trauma, onde foi observado um grande número de pacientes com diagnóstico de traumatismo cranioencefálico e que, ao passarem um tempo demasiado longo hospitalizados, evoluíam para a traqueostomia e, em consequência, pôde-se observar seu processo de desmame e decanulação que, no âmbito do Hospital Metropolitano é realizada de forma diferenciada. Foi estudada a relação entre o tempo de desmame e decanulação da traqueostomia e o tempo de hospitalização, a presença de complicações póstraqueostomia e o protocolo de desmame de traqueostomia adotado pelo hospital. Objetivamos analisar a relação entre o tempo de hospitalização dos pacientes vítimas de traumatismo cranioencefálico e que realizaram traqueostomia e a realização do protocolo de desmame do hospital, bem como observar a incidência das complicações que podem ocorrer. Os objetivos foram alcançados mediante realização de coleta de dados em prontuários de pacientes hospitalizados no HMUE no ano de 2012. Observou-se a correlação no tempo de desmame e decanulação de traqueostomia no tempo de alta hospitalar e no tempo de hospitalização do paciente vítima de Trauma Cranioencefálico. A não padronização no processo do cuidado com a traqueostomia causa um grande desentendimento entre vários profissionais na hora de lidar com esse tipo de situação.
\end{abstract}

Palavras-Chave: Trauma Craniano.Traqueostomia.Desmame.Fisioterapia.Decanulação

\footnotetext{
ABSTRACT: This research arose from the experience in a Hospital's Trauma, where a large number of patients with traumatic brain injury was observed and that by spending too long a time hospitalized, progressed to tracheostomy, and consequently, we could observe your processor of decannulation that, in the Hospital Metropolitano is performed differently. We studied the relationship between the time of weaning and decannulation of tracheostomy and 
hospitalization, the presence of post - tracheostomy complications and tracheostomy weaning protocol adopted by the hospital. It was purpose in research examining the relationship between duration of hospitalization of patients suffering from traumatic brain injury who underwent tracheostomy and completion of the weaning protocol of the hospital, and to observe the incidence of complications that can occur. The objectives were achieved by conducting data collection from medical records of patients hospitalized in HMUE in 2012. There was a correlation in weaning time and decannulation of tracheostomy with hospital stay of the patient with traumatic brain injury. The lack of standardization in the care process with tracheostomy causes a big misunderstanding between different healthy professionals when dealing with this kind of situation.

Keywords: Craniocerebral trauma. Tracheostomy. Weaning. Physiotherapy.Decannulation

\section{Introdução}

A traqueostomia é um procedimento utilizado com muita frequência nos hospitais gerais para o tratamento de insuficiência respiratória por muitas razões. ${ }^{1,2}$ Há regras específicas de indicação, mas não há regras determinantes para sua retirada ${ }^{3}$. É tida na maioria dos serviços como um procedimento eletivo e preventivo da sequela laríngea consequente da intubação traqueal prolongada translaríngea, além de estar relacionada precocemente à boa evolução intrahospitalar ${ }^{4}$.

Mendes $e$ cols. ${ }^{1}$ relatam que, na maioria dos hospitais, os fisioterapeutas são responsáveis pelo processo de desmame e decanulação. A interação entre o médico, o fisioterapeuta, o enfermeiro e o fonoaudiólogo permite diminuir o tempo de uso da traqueostomia, tornando-o mais seguro para o paciente, com menor risco de insucesso e complicações ${ }^{5}$.

Especificamente em pacientes com lesão neurológica grave, a realização precoce da prótese traqueal pode trazer benefícios, pois permite que o paciente possa sair mais rapidamente da ventilação mecânica e consequentemente da Unidade de Terapia Intensiva $^{6,7}$. Nesses casos, é frequentemente indicada pois facilita a remoção de secreções pulmonares, diminui o desconforto com a via aérea artificial e aumenta a mobilidade do paciente no leito $^{8}$. Esses benefícios possibilitam a redução do tempo de ventilação artificial, da incidência de pneumonia e do tempo de internação hospitalar. No entanto não há consenso na literatura quanto aos benefícios da traqueostomia em pacientes com Trauma Craniano 9 .

O período de desmame da traqueostomia consiste no tempo em que o paciente passa da situação de dependência da prótese traqueal para uma situação de independência da mesma, apresentando vias aéreas superiores com pérvias, sendo necessário pouco ou nenhum suporte ${ }^{10}$.

É baixo o número de estudos publicados acerca do processo de desmame da traqueostomia, principalmente envolvendo um protocolo validado de decanulação ${ }^{11}$. Portanto, o motivo da realização do estudo foi a importância a realização de pesquisas abordando este procedimento e seu consequente impacto no processo de hospitalização do paciente para que possam 
ser corrigidas prováveis falhas e favorecer uma melhor evolução clínica do indivíduo.

Objetivou-se avaliar o impacto do protocolo de desmame utilizado no Hospital Metropolitano nos pacientes com Trauma craniano, bem como observar variáveis demográficas, como sexo e idade, mais prevalentes.

\section{Método}

O estudo caracterizou-se como uma investigação retrospectiva, analíticadescritiva, sem intervenção, com coleta de dados em prontuários de pacientes maiores de 18 anos, com diagnóstico de Trauma Craniano, submetidos a traqueostomia eletiva, hospitalizados entre o período de Janeiro a Dezembro de 2012. Não poderiam ter sido submetidos a cricotiroidostomia prévia, sem associação a Trauma Raquimedular, não poderiam ter evoluído a óbito e nem terem sido transferidos para outro estabelecimento de saúde. O estudo foi aprovado pelo Comitê de Ética e Pesquisa da Universidade do Estado do Pará (UEPA), Belém, PA (Parecer $\mathrm{n}^{\circ}$ 336.119) e foi assinado o Termo de Consentimento para a Utilização de Dados para iniciar a pesquisa.

\section{Participantes}

Foram analisados 830 prontuários. Destes, 77 pacientes que preenchiam os critérios de inclusão foram selecionados. Posteriormente foram divididos em 03 grupos e submetidos a análise estatística descritiva e inferencial. Essa divisão deu-se pelo fato de que, ao serem analisados os dados, observou-se que alguns pacientes, apesar de terem feito uso da traqueostomia, não se enquadravam no protocolo de desmame e decanulação utilizado pelo hospital. Alguns, inclusive, utilizando-se de cânula de traqueostomia metálica ao longo de sua permanência hospitalar. O Grupo 1 foi constituído pelos pacientes que evoluíram dentro do protocolo do hospital, com oclusão da traqueostomia por 03 dias; o protocolo de desmame do Hospital Metropolitano consiste na oclusão da cânula de PVC em detrimento da troca para prótese metálica com diminuição progressiva do calibre da mesma, após a avaliação da equipe multiprofissional responsável pelo paciente. O tempo de oclusão da cânula de PVC dura, em média, 03 dias, sendo a decanulação realizada pelo médico responsável pelo doente. O Grupo 2 foi constituído pelos pacientes que evoluíram, por alguma razão, para substituição da cânula de PVC para metálica e o Grupo 3 foi composto por pacientes que foram submetidos ao protocolo de oclusão da cânula plástica, porém com um período maior que 03 dias.

\section{Instrumentos}

Os dados foram catalogados em uma planilha do Microsoft Excel 2013® e os Grupos foram submetidos a análise estatística 
descritiva e inferencial através do software BioEstat versão 5.3®.

\section{Análise Estatística}

Inicialmente as variáveis foram submetidos ao Teste de Lilliefors para análise da normalidade dos dados, tendo todas essas variáveis distribuição anormal. Posteriormente, a variável nominal sexo foi categorizada para que se pudesse ser executada as estatísticas descritiva.

Para análise da influência do protocolo de desmame no tempo de utilização de traqueostomia utilizou-se a Correlação de Pearson aceitando-se um nível de significância de $\mathrm{p}<0,05$, entre as variáveis Tempo de Hospitalização (Thosp); Tempo de traqueostomia (TTQT); Tempo de alta após a decanulação (Talta) e Tempo de Oclusão (Toclusão).

\section{Resultados}

Do total de participantes incluídos, 67 $(87,01 \%)$ eram do sexo masculino e 10 $(12,09)$ do sexo feminino com idade média de $34,14 \pm 14,03$.

$\mathrm{Na}$ tabela 1 podem ser encontrados os dados descritivos da amostra estudada no Grupo 1:

Tabela 1: Dados descritivos das variáveis na amostra estudada Grupo $1(n=48)$.

\begin{tabular}{cccccc}
\hline & $\begin{array}{c}\text { Idade } \\
\text { (anos) }\end{array}$ & $\begin{array}{c}\text { Tempo de } \\
\text { hospitalização (dias) }\end{array}$ & $\begin{array}{c}\text { Tempo de } \\
\text { traqueostomia (dias) }\end{array}$ & $\begin{array}{c}\text { Tempo de } \\
\text { oclusão (dias) }\end{array}$ & $\begin{array}{c}\text { Tempo de } \\
\text { alta (dias) }\end{array}$ \\
\hline Mínimo & 18 & 16 & 7 & 1 & 1 \\
Máximo & 76 & 219 & 142 & 3 & 72 \\
$\begin{array}{c}\text { Média } \\
\text { Aritmética }\end{array}$ & 35.4 & 42.3 & 27.2 & 2.22 & 7.60 \\
Desvio Padrão & 15.2 & 30.3 & 21.6 & 0.69 & 10.9 \\
\hline
\end{tabular}

Fonte: autor

Para o Grupo 1, observou-se que a idade variou entre 18 e 76 anos, com uma média de 35.4 anos. Com relação ao tempo de hospitalização, obteve-se um tempo de 16 a 219 dias, com uma média de 42.3 dias de hospitalização. No que diz respeito ao tempo de traqueostomia, a média foi de 27,25 dias, sendo um mínimo de 7 dias e um máximo de 142 de uso da prótese.
Relacionado ao tempo de oclusão, observou-se uma média de 2,2 dias, variando entre 01 e 03 dias. O tempo de alta do paciente após a decanulação foi em média de 7,6 dias com variação de 01-72 dias.

A tabela 2 traz dados a respeito do Grupo 2: 
Tabela 2: Dados descritivos das variáveis na amostra estudada Grupo 2 ( $n=7)$.

\begin{tabular}{cccccc}
\hline & Idade (anos) & $\begin{array}{c}\text { Tempo de } \\
\text { hospitalização } \\
\text { (dias) }\end{array}$ & $\begin{array}{c}\text { Tempo de } \\
\text { traqueostomia } \\
\text { (dias) }\end{array}$ & $\begin{array}{c}\text { Tempo de } \\
\text { oclusão (dias) }\end{array}$ & $\begin{array}{c}\text { Tempo de alta } \\
\text { (dias) }\end{array}$ \\
\hline Mínimo & 23 & 32 & 17 & 1 & 28 \\
Máximo & 50 & 169 & 70 & 8.2 & 9.8 \\
$\begin{array}{c}\text { Média } \\
\text { Aritmética }\end{array}$ & 35 & 83.5 & 42.7 & 11.1 & 7.79 \\
$\begin{array}{c}\text { Desvio Padrão } \\
\text { Tamanho da }\end{array}$ & 10.3 & 46.4 & 22.7 & 5 & 5 \\
amostra & 7 & 7 & 7 & & 5 \\
\hline
\end{tabular}

Fonte: autor

Do total de paciente $(\mathrm{n}=7), 02$ pacientes receberam alta hospitalar com traqueostomia metálica, devido a complicações (um com estenose subglótica e outro com estenose traqueal). Por este motivo, o tamanho da amostra em relação ao tempo de oclusão e ao tempo de alta após decanulação foi de 5 pacientes, uma vez que, ao receber alta hospitalar fazendo uso de traqueostomia, esses dois pacientes não realizaram as etapas de oclusão e decanulação.

Observou-se que, em relação ao Grupo 1, a variável faixa etária não sofreu grandes alterações já que o Grupo 2 apresentou uma média de 35 anos, com idades variando entre 23 e 50 anos. No que concerne a tempo de hospitalização, os pacientes do Grupo 2 apresentaram uma média de 83,5 dias de internação. Essa média é um pouco maior, quando comparada aos pacientes do Grupo 1, uma vez que o tempo de hospitalização pode estar relacionado a um tempo elevado de uso de tubo orotraqueal/traqueostomia ou a complicações e co-morbidades que o paciente apresentou.

A média de tempo de uso de cânula de PVC foi de 42,7 dias. O maior motivo encontrado para a troca da cânula de PVC para a metálica, durante a realização da pesquisa, foi a dificuldade no desmame da traqueostomia plástica, o que era revertido imediatamente após a troca para a metálica.

Dos 05 pacientes que conseguiram realizar a decanulação de traqueostomia metálica, observou-se uma média de 8,2 dias de oclusão até a decanulação, com variação entre 01 e 28 dias. Com relação ao tempo de alta hospitalar, os 05 pacientes que foram decanulados da traqueostomia metálica tiveram uma média de 9,8 dias para alta pós-decanulação, variando entre 01 e 20 dias.

A tabela 3 traz dados sobre os pacientes que, mesmo não realizando troca da prótese plástica para metálica, não se adequaram ao protocolo de desmame do HMUE, uma vez 
Tabela 3: Dados descritivos das variáveis na amostra estudada Grupo 3 ( $n=22)$.

\begin{tabular}{cccccc}
\hline & Idade (anos) & $\begin{array}{c}\text { Tempo de } \\
\text { hospitalização } \\
\text { (dias) }\end{array}$ & $\begin{array}{c}\text { Tempo de } \\
\text { traqueostomia } \\
\text { (dias) }\end{array}$ & $\begin{array}{c}\text { Tempo de } \\
\text { oclusão (dias) }\end{array}$ & $\begin{array}{c}\text { Tempo de alta } \\
\text { (dias) }\end{array}$ \\
\hline Mínimo & 18 & 19 & 9 & 4 & 12 \\
Máximo & 52 & 191 & 187 & 5.57 & 5.52 \\
$\begin{array}{c}\text { Média } \\
\text { Aritmética }\end{array}$ & 31 & 47.1 & 33 & 1.98 & 5.01 \\
$\begin{array}{c}\text { Desvio Padrão } \\
\text { Tamanho da } \\
\text { amostra }=\end{array}$ & 12.1 & 35.4 & 37.1 & 21 & 21 \\
\hline
\end{tabular}

Fonte: autor

Dos 22 pacientes que não se encaixaram no protocolo do Hospital, 01 recebeu alta com traqueostomia de PVC, uma vez que apresentou broncoaspiração recorrente, mas sem necessidade clínica de permanência no ambiente hospitalar, sendo este então encaminhado a um Serviço Especializado.

Com relação a faixa etária, a média encontrada foi de 31 anos, com variação entre 18 e 52 anos.

No que diz respeito ao tempo de hospitalização, a média em dias foi de 47,18 dias. O tempo médio de uso de traqueostomia para o Grupo 3 foi de 33 dias. O tempo de oclusão de traqueostomia para os pacientes do Grupo 3 foi de 5,57 dias, variando entre 04 e 12 dias. O tempo de alta hospitalar apresentou uma média de 5,52 dias, com variação de 0 a 20 dias, havendo somente um caso de paciente que recebeu alta no mesmo dia da decanulação.
Dentre o total de doentes $(n=77), 04$ pacientes evoluíram com complicações para não decanulação sendo essas: 02 pacientes com estenose, um sendo sub-glótica e outro com estenose de traquéia; um com laceração de traquéia; todas observadas mediante broncoscopia; e um com broncospiração.

A tabela 4 demonstra a influência do protocolo de desmame no tempo de utilização de traqueostomia, correlacionando as variáveis Tempo de Hospitalização (Thosp); Tempo de traqueostomia (TTQT); Tempo de alta após a decanulação (Talta) e Tempo de Oclusão (Toclusão). 
Tabela 4: Valores de correlação de Pearson entre as variáveis estudadas no Grupo 1.

\begin{tabular}{|c|c|c|c|c|c|}
\hline & $\begin{array}{l}\text { Thosp x } \\
\text { TTQT }\end{array}$ & $\begin{array}{l}\text { Thosp x } \\
\text { Toclusão }\end{array}$ & Thosp x Talta & $\begin{array}{l}\text { TTQT x } \\
\text { Toclusão Total }\end{array}$ & $\begin{array}{l}\text { Toclusão x } \\
\text { Talta }\end{array}$ \\
\hline $\mathrm{N}$ & 48 & 48 & 48 & 48 & 48 \\
\hline r (Pearson) & 0.9703 & -0.1153 & 0.8697 & 0.7431 & -0.1222 \\
\hline IC $95 \%$ & 0.95 a 0.98 & -0.39 a 0.17 & 0.78 a 0.93 & 0.58 a 0.85 & -0.39 a 0.17 \\
\hline IC $99 \%$ & 0.94 a 0.99 & -0.46 a 0.26 & 0.74 a 0.94 & 0.52 a 0.87 & -0.47 a 0.26 \\
\hline pvalor & $<0.0001 * * *$ & 0.435 & $<0.0001 * * *$ & $<0.0001 * * *$ & 0.4079 \\
\hline
\end{tabular}

Quando relacionamos o tempo de hospitalização com o tempo de uso de traqueostomia de PVC, obtemos um pvalor $<0,0001 * * *, \quad$ sendo então estatisticamente significante, mostrando que o tempo de utilização de traqueostomia influenciou no tempo de hospitalização do paciente.

Ao correlacionarmos o tempo de hospitalização ao tempo de alta hospitalar após a decanulação, o p-valor obtido foi menor que 0,0001 também obtendo significância estatística. E o tempo de traqueostomia correlacionado ao tempo de oclusão também obteve significância estatística, com p-valor $<0,0001$.

\section{Discussão}

Mendes $e$ cols. ${ }^{1}$, em uma pesquisa realizada com 21 pacientes sobre a proposta de um fluxograma para desmame de traqueostomia, observou que a faixa etária variava entre 20 e 89 anos.

Lima $e$ cols. $^{12}$, com 46 pacientes traqueostomizados e decanulados com sucesso, a média etária foi de 51,12 anos; investigação esta que diverge dos dados encontrados na presente pesquisa. Esse fato pode ser explicado, uma vez que a pesquisa dos autores acima citados foi realizada em um hospital geral, que engloba pacientes de diversas categorias e faixas etárias, a prevalência de pacientes idosos tende a ser maior. Já o Hospital Metropolitano de Urgência e Emergência, por ser um hospital referência em traumas têm em sua população uma grande quantidade de pacientes na faixa etária de adulto jovem.

Pasinie cols. ${ }^{9}$ realizaram uma pesquisa relacionando a influência da traqueostomia em pacientes vítimas de traumatismo 
cranioencefálico, observando que o tempo médio de hospitalização desses pacientes foi de 34,2 dias, sendo menor que a média de tempo de hospitalização observada na presente pesquisa.

$\mathrm{Na}$ pesquisa realizada por Lima e cols. ${ }^{12}$, acerca da influência da musculatura periférica no processo de decanulação de traqueostomia, aplicada em um hospital geral com 143 pacientes traqueostomizados, portadores de patologias em geral, onde 46 pacientes traqueostomizados que tiveram sucesso no desmame e decanulação da traqueostomia, o tempo médio do uso de traqueostomia foi de 27,11 dias, o que concorda com os dados encontrados nesta pesquisa.

No estudo de Mendes e cols. ${ }^{1}$, realizado para avaliar a proposta de um fluxograma para desmame de traqueostomia onde o protocolo de decanulação da traqueostomia inclui a troca da cânula de PVC para a cânula metálica, o tempo médio de oclusão foi de 20,94 dias com um mínimo de 04 e máximo de 87 dias para a decanulação do paciente, observando-se então diferença entre os resultados das duas pesquisas.

Não há na literatura nenhuma outra proposta de fluxograma de desmame e decanulação de traqueostomia ${ }^{13,14}$, muito menos onde preconiza-se um máximo de 03 dias para a decanulação do paciente, o que dificulta a realização de uma discussão mais aprofundada. Observou-se, porém, que os 48 pacientes encontrados no Grupo 1 obedeceram o tempo estipulado pelo protocolo, não apresentando complicações após decanulação.

Levando-se em conta a possibilidade de que os pacientes que evoluíram para a traqueostomia metálica apresentavam dificuldades no desmame da traqueostomia de PVC, observou-se um tempo relativamente pequeno entre o tempo de decanulação e a alta hospitalar, uma vez que, considerando-se essa dificuldade, o paciente não apresentava quaisquer outras restrições para a alta hospitalar, sendo protelada apenas pela demora no desmame.

A escassez de estudos acerca do tema tornam difícil a discussão mais detalhada sobre o tempo de alta do paciente após a decanulação ${ }^{15}$, porém observou-se um de tempo curto de internação após decanulação até a saída hospitalar do paciente. Este evento pode ter relação com o fato da traqueostomia ser uma porta de entrada para infecções ${ }^{16}$, culminando em uma permanência maior do paciente dentro do ambiente hospitalar.

Em uma pesquisa realizada por Lima, Marques e Toro $^{3}$, de 32 traqueostomias realizadas em pacientes portadores de patologias em geral, 13 pacientes $(40,62 \%)$ tiveram complicações como granuloma, malácea e estenose. Coelho e Stori ${ }^{17}$, realizaram uma pesquisa com 73 pacientes traqueostomizados, onde observaram a presença de um paciente com estenose laríngea $(3,3 \%)$. A estenose subglótica da 
laringe associada a granuloma e estenose traqueal são bastante frequentes ${ }^{18}$.

Entende-se que o tempo de hospitalização está relacionado ao tempo de alta após a decanulação e o tempo de traqueostomia também está correlacionado ao tempo de oclusão.

Lima, Marques e Toro ${ }^{3}$, optaram por realizar a decanulação após 60 dias de início do desmame no seu estudo, uma vez que dados prévios demonstraram que esse é o período crítico para o aparecimento de lesões secundárias a intubação e a traqueostomia.

O tema desmame e decanulação da traqueostomia é esquecido na grande maioria dos serviços e por grande parte dos profissionais da área da saúde. Ainda não há, atualmente, nenhum protocolo de desmame de traqueostomia validado, sendo cada serviço responsável pela elaboração do seu protocolo.

\section{Referencias}

1. ARANHA, S. C.; MATALOUN, S. E.; MOOK, M. Estudo Comparativo entre Traqueostomia Precoce e Tardia em Pacientes Sob ventilação Mecânica. Rev. Bras. Ter. Intensiva, v. 19, n. 4, out-dez, 2007.

2. CAMARGO, M. F. e cols. Análise das pressões intracuff em pacientes em terapia

\section{Considerações Finais}

Observou-se a correlação no tempo de desmame e decanulação de traqueostomia no tempo de alta hospitalar e no tempo de hospitalização do paciente vítima de Trauma Cranioencefálico. A não padronização no processo do cuidado com a traqueostomia causa um grande desentendimento entre vários profissionais na hora de lidar com esse tipo de situação. Cada um tem o seu ponto de vista e a sua opinião, e tudo torna-se uma verdadeira miscelânea de procedimentos e só quem sai perdendo nessa situação é o próprio paciente. Desta forma, este estudo serve de estopim para outros que virão. Deu-se o primeiro passo para um novo leque de opções de estudos que possam surgir para acrescentar mais conhecimentos para toda a comunidade acadêmica.

intensiva. Rev. Assoc. Med. Bras., v. 52, n. 6, p. 405-408, 2006.

3. COELHO, M. S.; STORI, W. S. Lesões Crônicas da laringe pela intubação traqueal. J. Pneumol., v. 21, n. 2, mar-abr, 2001.

4. GRILlO, H. C.; DONAHUE, D. M.; MATHISEN, D. J.; WAIN, J. C.; WRIGHT, C.D. Postintubation tracheal 
stenosis. Treatment and results. J. Thorac.

Cardiovasc. Surg., v. 109, n. 3, 1995

5. IBRAHIM, S. G. et al. Utilização de Equipamentos de Ventilação Não-Invasiva na Traqueostomia: Uma Alternativa para Alta na UTI? Rev. Bras. Ter. Intensiva, v. 24, n. 2, p. 167-172, 2012.

6. LEITE, A. G.; KUSSLER, D. Manejo da estenose traqueal distal recidivada por meio da endoprótese: relato de caso. J. Bras. Pneumol., v. 34, n. 2, p. 121-125, 2008.

7. LIMA, A. G.; MARQUES, A.; TORO, I. F. C. Sequelas pós-intubação e traqueostomia cirúrgica aberta: devemos sempre fazer a istmectomia? J. Bras. Pneumol., v. 35, n. 3, p. 227-233, 2009.

8. LIMA, C. A. e cols. Influência da força da musculatura periférica no sucesso da decanulação. Rev. Bras. Ter. Intensiva, v. 23 , n. 1 , p. $56,61,2011$

9. MANCEBO, S. B. C. et al. Estenosis traqueal isquemica: resultados deltratamientoquirurgico. Rev. Cubana Cir., v. 39, n. 1, p. 29-37, 2010.
10. MARTineZ, M. C. Q.; CUESTA, M. T. C. Alteraciones cognitivas después de um trauma craneoencefálico. Acta Colombiana de Psicologia, v. 14, n. 1, p. 71-80, 2011.

11. MENDES, F.; RANEA, P.; OLIVEIRA, A. C. T. Protocolo de desmame e decanulação de traqueostomia. Revista UNILUS Ensino e Pesquisa, v. 10, n. 20, jul/set, 2013.

12. MENDES, T. A. B.; CAVALHEIRO, L. V.; AREVALO, R. T.; SONEGHT, R. Estudo preliminar sobre a proposta de um fluxograma de decanulação em traqueostomia com atuação interdisciplinar. Einstein, v. 6, n. 1, p. 1-6, 2008.

13. ORTEGA, R. P. et al. Experiência Clínica em traqueotomia abierta. Rev. Otorrinolaringol. Cir. CabezaCuello, v. 71, p. 131-134, 2011.

14.PASINI, R. L. et al. A Influência da Traqueostomia Precoce no Desmame Ventilatório de Pacientes com Traumatismo Cranioencefálico Grave. Rev. Bras. Ter. Intensiva, v. 19, n. 2, abrjun, 2007. 
15.PINHEIRO, B. V.; TOSTES, R. O.; BRUM, C. I.; CARVALHO, E. V.; PINTO, S. P. S.; OlIVEIRA, J. C. A. Traqueostomia precoce versus traqueostomia tardia em pacientes com lesão cerebral aguda grave. J. Bras.

Pneumol., v. 36, n. 1, p. 84-91, 2010.

16.SAUERESSIG, M. G.; MACEDO, A. V.; ESPINEL，J. O.; EDELWEISS, M. I.; SANCHES, P. R. S.; XAVIER, R. G.
Modelo experimental de estenose traqueal mediante ressecção cirúrgica submucosa de anéis traqueais combinada com instilações de hidróxido de sódio. Rev. Col. Bras. Cir., v. 38, n. 6, p. 412-416, 2011.

17. VIANNA, A. Traqueostomia em pacientes sob ventilação mecânica: quando indicar? J. Bras. Pneumol., v. 33, n. 6, 2007. 